\title{
APPROXIMATIONS FOR THE LATE COEFFICIENTS IN ASYMPTOTIC EXPANSIONS ARISING IN THE METHOD OF STEEPEST DESCENTS
}

\author{
W. G. C. Boyd
}

\begin{abstract}
We set out two kinds of calculations for estimating the behavior of the late coefficients in the asymptotic expansions which arise in the method of steepest descents, Both are based on the reformulation of this method due to Berry and Howls. The first kind of calculation yields asymptotic expressions in which the higher-order terms diminish in inverse powers of $r$, while in the second kind of calculation, the higher-order terms diminish as in an inverse factorial series. We illustrate the calculations by applying them to the late coefficients in the asymptotic expansions of the Airy function, the modified Bessel function, and the gamma function.
\end{abstract}

\section{Introduction}

The purpose of this paper is to consider the behavior of the late coefficients in the asymptotic expansions that result from an application of the method of steepest descents. (The adjective "late" signifies that $r \gg 1$ where the coefficients are denoted by $a_{r}$, say.) It is well known that the behavior of the early coefficients may be described directly in terms of the local properties of the integrand at the saddle point through which the steepest-descents path passes. We shall find that the behavior of the late coefficients may be described in terms of the local properties of the integrand at certain other saddle points, the so-called adjacent saddle points. We shall present two kinds of results: in the first, the behavior of the late coefficients is represented by an expansion in inverse powers of $r$; and in the second, by an inverse factorial series. In both cases, we shall exploit the reformulation of the method of steepest descents due to Berry and Howls [2]. We shall find it convenient to refer to the exposition of the Berry-Howls approach given in $[5, \S 2]$.

Consider how the method of steepest descents would be used to find the asymptotic expansion, as $|z| \rightarrow \infty$, of the integral

$$
\int \mathrm{e}^{-z p(w)} q(w) \mathrm{d} w
$$

defined over some contour in the $w$-plane. The first stage in applying the method is to locate the zeros of $p^{\prime}(w)$ (more usually referred to as saddle points or stationary points in this context), and then to deform the contour to a path of steepest descents through one of the saddle points. We shall assume that $p(w)$ and $q(w)$ are holomorphic functions and that $p^{\prime}(w)$ has only simple zeros, located at $w^{(1)}, w^{(2)}, \ldots$, and suppose that the contour of integration is deformed into the path of steepest descents through the saddle point $w^{(n)}$. We thus are led to consider the asymptotic expansion of the

Received August 3, 1994, revised July 14, 1995.

1991 Mathematics Subject Classification, 41A60, 41A80.

Key words and phrases: steepest descents, asymptotic expansions, special functions. 
integral

$$
S^{(n)}(z)=z^{\frac{1}{2}} \int_{C^{(n)}(\theta)} \mathrm{e}^{-z\left[p(w)-p^{(n)}\right]} q(w) \mathrm{d} w
$$

where

$$
p^{(n)}=p\left(w^{(n)}\right)
$$

$C^{(n)}(\theta)$ is the path of steepest descents through the saddle point $w^{(n)}$ with the orientation specified in any particular problem, and $\theta$ denotes $\operatorname{ph}(z)$.

In a direct application of the method of steepest descents, the integral in (2) is transformed to a canonical form to which Watson's lemma can be applied [13, p. 89]. One thus finds that $S^{(n)}(z)$ enjoys the asymptotic expansion

$$
S^{(n)}(z) \sim \sum_{r=0}^{\infty} \frac{a_{r}}{z^{r}}
$$

where the values of the coefficients $a_{r}$ are expressed in terms of the local properties of $p(w)$ and $q(w)$ at $w=w^{(n)}$. In this approach, the leading coefficients are found to be

$$
\begin{aligned}
& a_{0}=\sqrt{\frac{2 \pi}{p^{\prime \prime}}} q \\
& a_{1}=\sqrt{\frac{2 \pi}{p^{\prime \prime}}} \frac{5 p^{\prime \prime \prime}{ }^{2} q-3 p^{\prime \prime} p^{\prime \prime \prime \prime} q-12 p^{\prime \prime} p^{\prime \prime \prime} q^{\prime}+12 p^{\prime \prime 2} q^{\prime \prime}}{24 p^{\prime \prime}},
\end{aligned}
$$

where the various derivatives of $p$ and $q$ are evaluated at the saddle point $w=w^{(n)}$. The expressions for higher coefficients are successively more complicated. (Dingle [9, pp. 119-121] gives expressions for the coefficients up to and including $a_{4}$.)

In the reformulation of the method of steepest descents by Berry and Howls [2], the coefficients $a_{r}$ are represented by

$$
a_{r}=\frac{\Gamma\left(r+\frac{1}{2}\right)}{2 \pi \mathrm{i}} \oint_{\Gamma^{(n)}} \frac{q(w)}{\left[p(w)-p^{(n)}\right]^{r+\frac{1}{2}}} \mathrm{~d} w
$$

where $\Gamma^{(n)}$ is a closed contour surrounding the saddle point $w^{(n)}$, traversed counterclockwise. (This representation had been given earlier by Copson $[7$, p. 69] and Dingle $[9$, p. 119].) We shall discuss the precise specification of the integral (6) in $\S 2$. The integrand in (6) alternatively may be expressed in the form (1), that is,

$$
a_{r}=\frac{\Gamma\left(r+\frac{1}{2}\right)}{2 \pi \mathrm{i}} \oint_{\Gamma^{(n)}} \exp \left(-\left(r+\frac{1}{2}\right) \ln \left(p(w)-p^{(n)}\right)\right) q(w) \mathrm{d} w .
$$

It is natural to enquire whether techniques for the asymptotic evaluation of integralsand, in particular, the method of steepest descents itself-are applicable to the representation (7) when $r \gg 1$. That is to say, can the asymptotic behavior of the late coefficients (the behavior of $a_{r}$ as $r \rightarrow \infty$ ) be determined from (7)? We shall find that it can under a wide variety of circumstances which are described in $\S 2$. Berry and Howls [3, §2.1] show formally how integrals such as (7) may be evaluated asymptotically. In $\S 3$, we shall apply the results of $\S 2$ to three examples: the late coefficients arising in the asymptotic expansions of the Airy function, the modified Bessel function, and the gamma function.

The results of $\S 2$ and $\S 3$ express the behavior of the late coefficients as expansions in inverse powers of $r$. In $\S 4$, we shall show how a different sort of expression for the late coefficients - as inverse factorial series-may be determined. This approach uses a representation for the coefficients $a_{r}$ which differs from (6) or (7) above. Instead, 
starting with the fundamental result which Berry and Howls themselves exploited in deriving their hyperasymptotic scheme [2], we deduce an alternative representation, given in (52) below, from which inverse factorial series arise naturally. In an earlier paper [6], we showed how this approach could be applied to the late coefficients in the asymptotic expansion of the gamma function; in $\S 4$, we shall show how it may be used for the Airy function and modified Bessel function. Dingle [9] gives many formal results using inverse factorial series (in particular, see Chapter VII, which discusses late terms).

We conclude in $\S 5$ with a short discussion. An appendix demonstrates the equivalence of the representation (39) for the gamma function and an apparently different one used in a similar context by Diekmann [8]. Diekmann's analysis, which was concerned specifically with the gamma function, foreshadows in some respects the approach we set out in $\S 2$ and $\S 3$.

\section{The asymptotic behavior of the late coefficients}

The starting point for our analysis will be the representation (7) for the coefficients $a_{r}$. But first, it is necessary to set out conditions on the functions $p(w)$ and $q(w)$, and to specify the branches in the integrands of (6) and (7).

The conditions we shall require of $p(w)$ and $q(w)$ are closely adapted from those given in [5, p. 501]:

\section{Conditions 2.1}

(i) The functions $p(w)$ and $q(w)$ are analytic at every point in the closure of a domain $\Delta^{(n)}$.

(ii) There is exactly one (interior) point of $\Delta^{(n)}$ which is a saddle point of $p(w)$. At this point, $w^{(n)}, p^{\prime \prime}\left(w^{(n)}\right) \neq 0$.

(iii) Any point $w$ is in the closure of $\Delta^{(n)}$ if and only if it can be reached by exactly one path of steepest descents,

$$
\operatorname{ph}\left(p(w)-p^{(n)}\right)=\text { constant }
$$

emanating from $w^{(n)}$.

(iv) The boundary of $\Delta^{(n)}$ in the finite $w$-plane comprises the union of the adjacent contours $C^{(m)}$, each of which contains exactly one adjacent saddle point $w^{(m)}$ of $p(w)$ and on which

$$
\operatorname{ph}\left(p(w)-p\left(w^{(m)}\right)\right)=\operatorname{ph}\left(p^{(n m)}\right) .
$$

(The right-hand side is defined in (8) below.) Furthermore, $p^{\prime \prime}\left(w^{(m)}\right) \neq 0$.

(v) As $|w| \rightarrow \infty$ in $\Delta^{(n)},[p(w)]^{-r-\frac{1}{2}} q(w)=\mathrm{o}\left(w^{-1}\right)$; moreover, $[p(w)]^{-r-\frac{1}{2}} q(w) \rightarrow 0$ sufficiently rapidly so that

$$
\int_{C^{(m)}}\left|\frac{q(w) \mathrm{d} w}{[p(w)]^{r+\frac{1}{2}}}\right|
$$

exists for each adjacent contour $C^{(m)}$.

In condition (iv), the right-hand side of the equation is defined by

$$
p^{(n m)}=p^{(m)}-p^{(n)}
$$




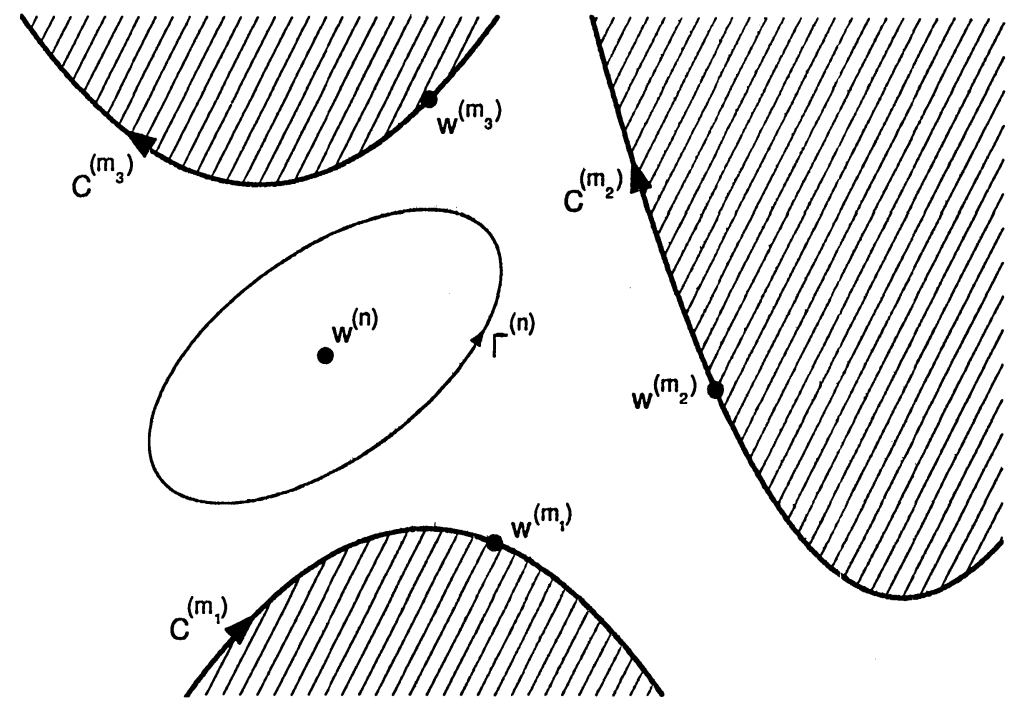

FIgURE 1. A typical domain $\Delta^{(n)}$ associated with the saddle point $w^{(n)}$, in which Conditions 2.1 apply. (The domain $\Delta^{(n)}$ is shown unshaded.) The contour $\Gamma^{(n)}$, which is traversed anticlockwise, encloses the saddle point $w^{(n)}$ within the domain $\Delta^{(n)}$. The contours on the boundary of the domain-the figure depicts $C^{\left(m_{1}\right)}, C^{\left(m_{2}\right)}$, $C^{\left(m_{3}\right)}$, passing through the adjacent saddle points $w^{\left(m_{1}\right)}, w^{\left(m_{2}\right)}$, $w^{\left(m_{3}\right)}$, respectively-are the adjacent contours.

We make a number of observations about Conditions 2.1 ${ }^{1}$. A typical domain $\Delta^{(n)}$ is illustrated in Figure 1. The term adjacent contour was introduced in [5], following Berry and Howls' term adjacent saddle [2]: the saddle point $w^{(m)}$ of $p(w)$ is adjacent to $w^{(n)}$ if it can be reached from $w^{(n)}$ by means of a path of steepest descents given by condition (iii) above; condition (iv) above implicitly defines the corresponding adjacent contour. We remark that although Conditions 2.1 refer to $p(w)$ itself rather than to the function $\ln \left(p(w)-p^{(n)}\right)$ which appears in (7), the conditions are indeed appropriate, as we shall show below.

Next we consider the precise specifications for the integrands of (6) and (7). The specification of the square root $\left[p(w)-p^{(n)}\right]^{\frac{1}{2}}$ in the integrand of $(6)$ is discussed in detail in [5, pp. 498-499]: we require $\left[p(w)-p^{(n)}\right]^{\frac{1}{2}}$ to be positive for those values of $w$ which lie on the half of the contour $C^{(n)}(0)$ which is traversed after the saddle point $w^{(n)}$ (referred to as $C_{+}^{(n)}$ with $\theta=0$ in [5]), and define it elsewhere by analytic continuation. Note that because $p(w)-p^{(n)}$ has a double zero at $w=w^{(n)}$, the integrand of (6) is actually single-valued. Consistently with this specification of the square root in the integrand of (6), we define the logarithm in (7) to have zero imaginary part for those values of $w$ which lie on the half of the contour $C^{(n)}(0)$ which is traversed after the saddle point $w^{(n)}$, and define it elsewhere by analytic continuation. Although the logarithm in (7) is not itself single-valued, the integrand is, so it is not necessary to

\footnotetext{
${ }^{1}$ There is an omission in the conditions given by $[5$, p. 501]; the requirement that $[p(w)]^{-r-\frac{1}{2}} q(w)=\mathrm{o}\left(w^{-1}\right)$ as $|w| \rightarrow \infty$ in $\Delta^{(n)}$, which is given in condition (v) above, also is necessary in [5].
} 
consider branch cuts or other devices to ensure that the contour integral in (7) is well defined.

Now consider how to exploit the representation (7) to find the asymptotic behavior of the late coefficients $a_{r}$. By virtue of Conditions 2.1, we may deform the contour of integration $\Gamma^{(n)}$ out to the boundary of $\Delta^{(n)}$, and thus obtain

$$
a_{r}=\frac{\Gamma\left(r+\frac{1}{2}\right)}{2 \pi \mathrm{i}} \sum_{m} \int_{C^{(m)}} \exp \left(-\left(r+\frac{1}{2}\right) \ln \left(p(w)-p^{(n)}\right)\right) q(w) \mathrm{d} w,
$$

in which $a_{r}$ is represented as a sum of integrals over the adjacent contours (cf. [3, equation (15)]). Consider how the method of steepest descents may be applied to this integral for $r \rightarrow \infty$. First, the stationary points of $\ln \left(p(w)-p^{(n)}\right)$ have to be located. These occur at the zeros of $p^{\prime}(w)$, that is, they coincide with the saddle points of $p(w)$. On each contour $C^{(m)}$ in (9), therefore, there is exactly one stationary point. The second stage in the method of steepest descents is to factor out the value of the exponential in each integrand of (9), and then to determine the paths of steepest descents from each saddle point. We thus find

$$
a_{r}=\frac{\Gamma\left(r+\frac{1}{2}\right)}{2 \pi \mathrm{i}} \sum_{m} \frac{1}{\left[p^{(n m)}\right]^{r+\frac{1}{2}}} \int_{C^{(m)}} \exp \left(-\left(r+\frac{1}{2}\right) \ln \left(\frac{p(w)-p^{(n)}}{p^{(n m)}}\right)\right) q(w) \mathrm{d} w .
$$

The square roots $\left[p^{(n m)}\right]^{\frac{1}{2}}$ in this expression are special cases of the square root of $\left[p(w)-p^{(n)}\right]^{\frac{1}{2}}$, discussed in the previous paragraph; a fortiori, they are defined without ambiguity. For each value of $m$ in (10), the argument of the logarithm is real and greater than or equal to 1 for values of $w$ on $C^{(m)}$ : it follows, therefore, that the adjacent contours are paths of steepest descents for the integrands in (10).

Therefore, the method of steepest descents may be applied directly to the integrals in (10) to yield, at leading order,

$$
\int_{C^{(m)}}=\sqrt{\frac{2 \pi}{r+\frac{1}{2}}} \frac{\left[p^{(n m)}\right]^{\frac{1}{2}}}{\left[p^{\prime \prime}\left(w^{(m)}\right)\right]^{\frac{1}{2}}} q\left(w^{(m)}\right)\left[1+\mathrm{O}\left(\frac{1}{r}\right)\right]
$$

as $r \rightarrow \infty$. The correct branch of the square root $\left[p^{\prime \prime}\left(w^{(m)}\right)\right]^{\frac{1}{2}}$ in (11) needs to be specified carefully. Note that, as we remarked above, the argument of the logarithm in (10) is real, and also that as $r \rightarrow \infty$ the value of the integral in (10) is increasingly dominated by the local behavior of the integrand in the neighborhood of the saddle point $w=w^{(m)}$. Thus, one infers that

$$
\operatorname{ph}\left(\left[p^{(n m)}\right]^{\frac{1}{2}}\right)-\operatorname{ph}\left(\left[p^{\prime \prime}\left(w^{(m)}\right)\right]^{\frac{1}{2}}\right)
$$

must equal the angle which the tangential direction vector at $w=w^{(m)}$ makes with the positive real axis (with account taken of the sense in which the contour $C^{(m)}$ is traversed). The square root $\left[p^{\prime \prime}\left(w^{(m)}\right)\right]^{\frac{1}{2}}$ in (11), therefore, must be specified by choosing that value which enables requirement (12) to be satisfied. (Examples are provided in $\S 3$.) With $\left[p^{\prime \prime}\left(w^{(m)}\right)\right]^{\frac{1}{2}}$ thus specified, we may assert that

$$
a_{r}=-\mathrm{i} \frac{\Gamma\left(r+\frac{1}{2}\right)}{\sqrt{2 \pi\left(r+\frac{1}{2}\right)}} \sum_{m} \frac{q\left(w^{(m)}\right)}{\left[p^{(n m)}\right]^{r}\left[p^{\prime \prime}\left(w^{(m)}\right)\right]^{\frac{1}{2}}}\left[1+\mathrm{O}\left(\frac{1}{r}\right)\right]
$$

as $r \rightarrow \infty$.

For some of the applications that we shall consider later we shall need also the next terms in the expansions appearing in (13), and, for this purpose, we may appeal to 
the formula for $a_{1}$ given in (5). Now the second, third, and fourth derivatives of the logarithm in (10), respectively, have the values

$$
\frac{p^{\prime \prime}\left(w^{(m)}\right)}{p^{(n m)}}, \quad \frac{p^{\prime \prime \prime}\left(w^{(m)}\right)}{p^{(n m)}}, \quad \frac{p^{\prime \prime \prime \prime}\left(w^{(m)}\right)}{p^{(n m)}}-3\left[\frac{p^{\prime \prime}\left(w^{(m)}\right)}{p^{(n m)}}\right]^{2}
$$

at $w=w^{(m)}$, so the final factor in (13) -in the square brackets-is

$$
1+\frac{9 p^{\prime \prime}{ }^{3} q+p^{(n m)}\left\{5 p^{\prime \prime \prime}{ }^{2} q-3 p^{\prime \prime} p^{\prime \prime \prime \prime} q-12 p^{\prime \prime} p^{\prime \prime \prime} q^{\prime}+12 p^{\prime \prime 2} q^{\prime \prime}\right\}}{24 p^{\prime \prime} q} \frac{1}{r}+\mathrm{O}\left(\frac{1}{r^{2}}\right)
$$

where the various derivatives of $p$ and $q$ are evaluated at the adjacent saddle points $w=w^{(m)}$. In principle, higher-order terms than those given in (15) could be found, but, as we noted in $\S 1$, these become successively more complicated. However, it is worth remarking on the form of the higher-order terms, in inverse powers of $r$. Later, in $\S 4$, we shall find approximations of a different kind, as inverse factorial series.

The description of the behavior of the coefficient $a_{r}$ as $r \rightarrow \infty$ given by (13) may be slightly simplified by an appeal to the result

$$
\frac{\Gamma\left(r+\frac{1}{2}\right)}{\sqrt{r+\frac{1}{2}}}=\Gamma(r)\left(1-\frac{3}{8 r}+\mathrm{O}\left(\frac{1}{r^{2}}\right)\right)
$$

as $r \rightarrow \infty[11$, p. 119]. Therefore, we find that (13) becomes

$$
a_{r}=-\mathrm{i} \frac{\Gamma(r)}{\sqrt{2 \pi}} \sum_{m} \frac{q\left(w^{(m)}\right)}{\left[p^{(n m)}\right]^{r}\left[p^{\prime \prime}\left(w^{(m)}\right)\right]^{\frac{1}{2}}}\left[1+\mathrm{O}\left(\frac{1}{r}\right)\right] .
$$

The next terms in the expansions appearing in (17) can be found directly from (15) and (16); one finds that the final factor in (17) - in the square brackets - is

$$
1+p^{(n m)} \frac{5{p^{\prime \prime \prime}}^{2} q-3 p^{\prime \prime} p^{\prime \prime \prime \prime} q-12 p^{\prime \prime} p^{\prime \prime \prime} q^{\prime}+12{p^{\prime \prime}}^{2} q^{\prime \prime}}{24 p^{\prime \prime} q} \frac{1}{r}+\mathrm{O}\left(\frac{1}{r^{2}}\right)
$$

where the various derivatives of $p$ and $q$ are evaluated at the adjacent saddle points $w=w^{(m)}$.

The factors $\left[p^{(n m)}\right]^{r}$, which appear in (17), can be expected to differ in magnitude for the various adjacent saddle points. In particular, if there is a value of $m$, say $\widehat{m}$, for which the value of $\left|p^{(n \widehat{m})}\right|$ is less than $\left|p^{(n m)}\right|$ for all the other adjacent saddles, then

$$
a_{r} \sim \frac{-\mathrm{i} q\left(w^{(\widehat{m})}\right)}{\sqrt{2 \pi}\left[p^{\prime \prime}\left(w^{(\widehat{m})}\right)\right]^{\frac{1}{2}}} \frac{\Gamma(r)}{\left[p^{(n \widehat{m})}\right]^{r}}
$$

as $r \rightarrow \infty$. The constant factor aside, this is in the form of 'a factorial divided by a power', a result we shall return to in $\S 5$. However, when the least value of $\left|p^{(n m)}\right|$ is shared by two or more adjacent saddles - as in the examples we shall consider in $\S 3.2$ and $\S 3.3$ - the leading-order behavior of $a_{r}$ may be more complicated. Indeed, for the example in $\S 3.3$ (the gamma function), we shall find that the even and odd late coefficients differ substantially in relative magnitude. 


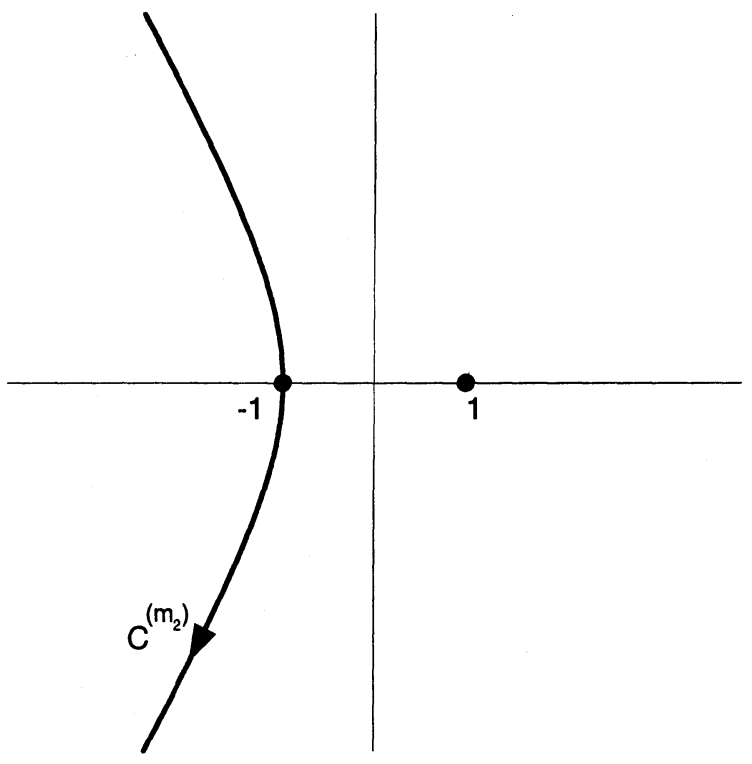

FIGURE 2. The domain $\Delta^{(1)}$ associated with the saddle point $w^{(1)}=$ 1 for the integral representation of the Airy function $\mathrm{Ai}\left(\left(\frac{3}{2} z\right)^{\frac{2}{3}}\right)$ considered in $\S 3.1$. There is only one adjacent contour, $C^{(2)}$.

\section{Examples}

3.1. The Airy function Ai. We shall find it convenient to consider the Airy function of argument $\left(\frac{3}{2} z\right)^{\frac{2}{3}}$, rather than $\operatorname{Ai}(z)$ itself. Its integral representation is $[13$, p. 90]

$$
\operatorname{Ai}\left(\left(\frac{3}{2} z\right)^{\frac{2}{3}}\right)=\frac{1}{2 \pi \mathrm{i}}\left(\frac{3}{2} z\right)^{\frac{1}{3}} \int_{C} e^{-z p(w)} \mathrm{d} w
$$

where

$$
p(w)=-\frac{1}{2} w^{3}+\frac{3}{2} w .
$$

In (20), and subsequently in this subsection, fractional powers of $z$ take their principal values for $|\theta|<\pi$; the contour $C$ runs from $\infty \exp \left(\mathrm{i} \frac{1}{3}(-\pi-\theta)\right)$ to $\infty \exp \left(\mathrm{i} \frac{1}{3}(\pi-\theta)\right)$. The function $p(w)$ has two saddle points, $w^{(1)}=1$ and $w^{(2)}=-1$. Following [5, p. 507], we shall consider the function $S^{(1)}(z)$, which is related to the Airy function by

$$
\operatorname{Ai}\left(\left(\frac{3}{2} z\right)^{\frac{2}{3}}\right)=-\mathrm{i} 2^{-\frac{4}{3}} 3^{\frac{1}{3}} \pi^{-1} z^{-\frac{1}{6}} \mathrm{e}^{-z} S^{(1)}(z),
$$

and which is defined by

$$
S^{(1)}(z)=z^{\frac{1}{2}} \int_{C^{(1)}} \mathrm{e}^{-z[p(w)-1]} \mathrm{d} w
$$

(The contour $C$ in (20) may be deformed to the path of steepest descents $C^{(1)}$ through the saddle point $w^{(1)}$ for $|\theta|<\pi$.) 
The asymptotic expansion of $S^{(1)}(z)$ is readily found from the well-known asymptotic expansion for the Airy function:

$$
S^{(1)}(z) \sim \sum_{r=0}^{\infty} \frac{a_{r}}{z^{r}}
$$

for $|\theta|<\pi$ where

$$
a_{r}=\mathrm{i}(-1)^{r} \sqrt{\frac{2 \pi}{3}} \frac{\Gamma\left(3 r+\frac{1}{2}\right)}{54^{r} r ! \Gamma\left(r+\frac{1}{2}\right)}
$$

(e.g., $[5$, p. 507]).

We shall illustrate the general calculation described in $\S 2$ for this example. It can easily be verified that Conditions 2.1 hold good with respect to the saddle point $w^{(1)}$ for all $r \geq 0$; there is only one adjacent saddle point, $w^{(2)}$, and the domain $\Delta^{(1)}$ comprises all the points to the right of the adjacent contour $C^{(2)}$. One readily finds that $p^{(12)}=-2$ and $p^{\prime \prime}\left(w^{(2)}\right)=3$; taking account of requirement (12) and the orientation of $C^{(2)}$ in Figure 2, one finds that $\operatorname{ph}\left(\left[p^{\prime \prime}\left(w^{(2)}\right)\right]^{\frac{1}{2}}\right)$ must satisfy, modulo $2 \pi$

$$
\frac{1}{2} \pi-\operatorname{ph}\left(\left[p^{\prime \prime}\left(w^{(2)}\right)\right]^{\frac{1}{2}}\right)=-\frac{1}{2} \pi
$$

that is, $\operatorname{ph}\left(\left[p^{\prime \prime}\left(w^{(2)}\right)\right]^{\frac{1}{2}}\right)=\pi$, modulo $2 \pi$. Hence, from (19),

$$
a_{r} \sim \frac{\mathrm{i}}{\sqrt{6 \pi}} \frac{\Gamma(r)}{(-2)^{r}}
$$

as $r \rightarrow \infty$. It can be shown by using Stirling's formula [11, p. 88] that the asymptotic behavior of the right-hand side of (25) for $r \rightarrow \infty$ indeed is given by (27).

3.2. The modified Bessel function $K_{\nu}$. The modified Bessel function $K_{\nu}(z)$ may be defined for $|\theta|<\frac{1}{2} \pi$ by

$$
K_{\nu}(z)=\frac{1}{2} \int_{-\infty}^{\infty} \mathrm{e}^{-z p(w)} q(w) \mathrm{d} w
$$

where

$$
p(z)=\cosh (w), \quad q(w)=\mathrm{e}^{\nu w}
$$

$\left[11\right.$, p. 250]. The function $p(w)$ has saddle points at $w^{(n)}=n \pi \mathrm{i}$ for $n=0, \pm 1, \pm 2, \ldots$, and, for $|\theta|<\frac{1}{2} \pi$, the contour of integration in (28) may be deformed to $C^{(0)}$, the path of steepest descents through the saddle point $w^{(0)}$. Following [5, p. 510], we shall consider the function $S^{(0)}(z)$, which is related to $K_{\nu}(z)$ by

$$
K_{\nu}(z)=\frac{1}{2} z^{-\frac{1}{2}} \mathrm{e}^{-z} S^{(0)}(z),
$$

and which is defined by

$$
S^{(0)}(z)=z^{\frac{1}{2}} \int_{C^{(0)}} \mathrm{e}^{-z[p(w)-1]} q(w) \mathrm{d} w .
$$

A standard appeal to analytic continuation shows that (31) is actually valid for a larger domain than we have indicated; it holds good for $|\theta|<\pi$.

Only two of the infinite number of saddle points are adjacent to $w^{(0)}$, namely, $w^{(1)}$ and $w^{(-1)}$ (see Figure 3). One readily verifies that Conditions 2.1 hold good with respect to the saddle point $w^{(0)}$ provided $r>|\operatorname{Re}(\nu)|-\frac{1}{2}$. Since we are considering $r \gg 1$, this proviso involves little loss of generality (unless $|\operatorname{Re}(\nu)|$ is very large, in which case different approximations are appropriate in any case). The domain $\Delta^{(0)}$ 


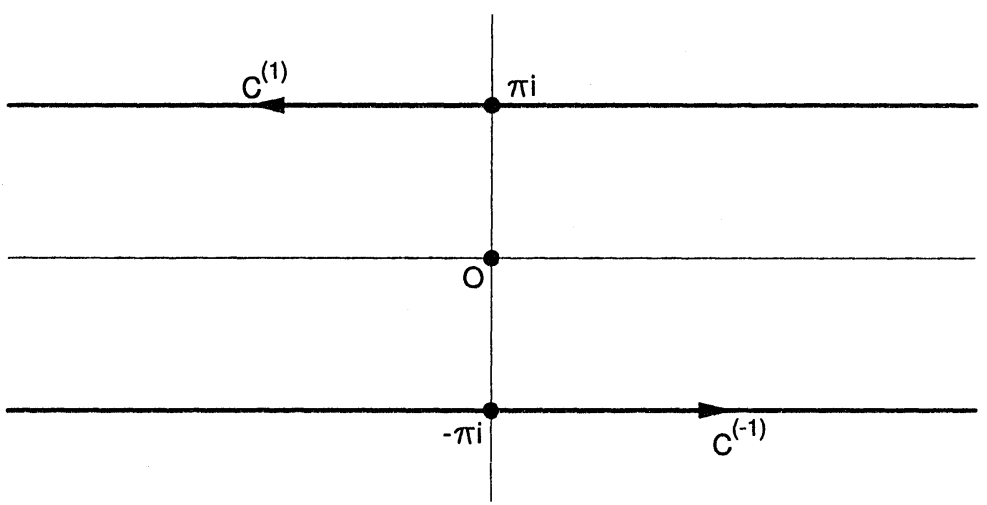

FIGURE 3. The domain $\Delta^{(0)}$ associated with the saddle point $w^{(0)}=$ 0 for the integral representation of the modified Bessel function $K_{\nu}(z)$ considered in $\S 3.2$. There are two adjacent contours, $C^{(-1)}$ and $C^{(1)}$.

is $|\operatorname{Im}(z)|<\pi$. One finds $p^{(01)}=-2, p^{\prime \prime}\left(w^{(1)}\right)=-1, q\left(w^{(1)}\right)=\mathrm{e}^{\nu \pi \mathrm{i}}, p^{(0,-1)}=-2$, $p^{\prime \prime}\left(w^{(-1)}\right)=-1$, and $q\left(w^{(-1)}\right)=\mathrm{e}^{-\nu \pi \mathrm{i}}$; taking account of requirement $(12)$ and the orientations of $C^{(1)}$ and $C^{(-1)}$ in Figure 3, one finds that, modulo $2 \pi$,

$$
\frac{1}{2} \pi-\operatorname{ph}\left(\left[p^{\prime \prime}\left(w^{(1)}\right)\right]^{\frac{1}{2}}\right)=\pi
$$

and

$$
-\frac{1}{2} \pi-\operatorname{ph}\left(\left[p^{\prime \prime}\left(w^{(-1)}\right)\right]^{\frac{1}{2}}\right)=0,
$$

that is, the square roots must be chosen so that

$$
\operatorname{ph}\left(\left[p^{\prime \prime}\left(w^{(1)}\right)\right]^{\frac{1}{2}}\right)=\operatorname{ph}\left(\left[p^{\prime \prime}\left(w^{(-1)}\right)\right]^{\frac{1}{2}}\right)=-\frac{1}{2} \pi,
$$

modulo $2 \pi$. Hence, from (17),

$$
a_{r}=\frac{\mathrm{e}^{\nu \pi \mathrm{i}}}{\sqrt{2 \pi}} \frac{\Gamma(r)}{(-2)^{r}}\left[1+\mathrm{O}\left(\frac{1}{r}\right)\right]+\frac{\mathrm{e}^{-\nu \pi \mathrm{i}}}{\sqrt{2 \pi}} \frac{\Gamma(r)}{(-2)^{r}}\left[1+\mathrm{O}\left(\frac{1}{r}\right)\right]
$$

as $r \rightarrow \infty$. The result (35) simplifies to

$$
a_{r}=\sqrt{\frac{2}{\pi}} \cos (\nu \pi) \frac{\Gamma(r)}{(-2)^{r}}\left[1+\mathrm{O}\left(\frac{1}{r}\right)\right],
$$

except possibly when $\nu=\frac{1}{2}, \frac{3}{2}, \frac{5}{2}, \ldots$. This latter possibility is, however, of no direct interest because it is known (see (37) below) that, for sufficiently large $r$ (and in particular for $r>\nu$ ), the coefficients $a_{r}=0$ for such values of $\nu$.

The coefficients $a_{r}$ are given exactly by $[4$, p. 242]

$$
a_{r}=\sqrt{\frac{2}{\pi}} \cos (\nu \pi) \frac{\Gamma\left(r+\frac{1}{2}+\nu\right) \Gamma\left(r+\frac{1}{2}-\nu\right)}{(-2)^{r} \Gamma(r+1)},
$$

with the understanding that the limiting form is taken for $\nu=\frac{1}{2}, \frac{3}{2}, \frac{5}{2}, \ldots$ when $r<\nu$. An appeal to Stirling's formula shows that the asymptotic behavior of the right-hand side of (37) for $r \rightarrow \infty$ indeed is given by (36). 


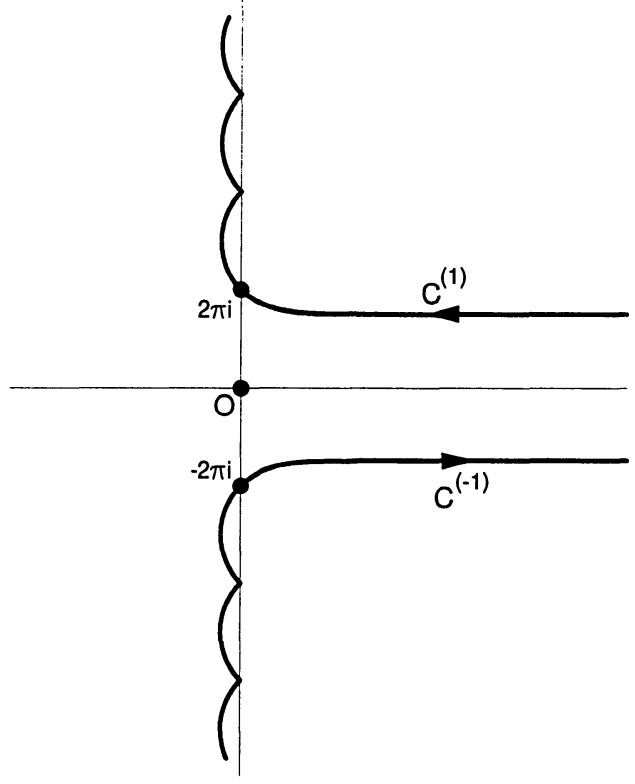

FiguRE 4. The domain $\Delta^{(0)}$ associated with the saddle point $w^{(0)}=$ 0 for the integral representation of the gamma function $\Gamma(z)$ considered in $\S 3.3$. There are two adjacent contours, $C^{(-1)}$ and $C^{(1)}$.

3.3. The gamma function. In discussing the gamma function, we follow the analysis of [6]. Our starting point is Euler's integral

$$
\Gamma(z)=\int_{0}^{\infty} \mathrm{e}^{-t} t^{z-1} \mathrm{~d} t
$$

which is appropriate for $|\theta|<\frac{1}{2} \pi$. The change of variable $w=\ln (t / z)$ transforms Euler's integral to

$$
\Gamma(z)=z^{z} \int_{-\infty}^{\infty} \mathrm{e}^{-z p(w)} \mathrm{d} w
$$

a form which is more suitable for our purpose, again appropriate for $|\theta|<\frac{1}{2} \pi$. Here, the function $p(w)$ is defined by

$$
p(w)=\mathrm{e}^{w}-w
$$

its saddle points are at $w^{(n)}=2 n \pi \mathrm{i}$ for $n=0, \pm 1, \pm 2, \ldots$. We consider the function $S^{(0)}(z)$, which is related to the gamma function by

$$
\Gamma(z)=z^{z-\frac{1}{2}} \mathrm{e}^{-z} S^{(0)}(z),
$$

and which is defined by

$$
S^{(0)}(z)=z^{\frac{1}{2}} \int_{C^{(0)}} \mathrm{e}^{-z[p(w)-1]} \mathrm{d} w
$$

for $|\theta|<\frac{1}{2} \pi$ (the definition differs slightly, by a factor of $\sqrt{2 \pi}$, from that of $[6]$ ).

In [6] we showed that one may regard only two of the saddle points $w^{(m)}$ as adjacent to $w^{(0)}$, namely $w^{(1)}$ and $w^{(-1)}$. The adjacent contours $C^{(1)}$ and $C^{(-1)}$ then pass through all the other saddle points for $m>0$ and $m<0$, respectively (see Figure 4). 
It may be verified that Conditions 2.1 hold good with respect to the saddle point $w^{(0)}$ provided $r \geq 1$; the domain $\Delta^{(0)}$ is illustrated in Figure 4. One finds $p^{(01)}=-2 \pi \mathrm{i}$, $p^{\prime \prime}\left(w^{(1)}\right)=1, p^{(0,-1)}=2 \pi \mathrm{i}$, and $p^{\prime \prime}\left(w^{(-1)}\right)=1$; taking account of requirement $(12)$ and the orientations of $C^{(1)}$ and $C^{(-1)}$ in Figure 4, one finds that, modulo $2 \pi$,

$$
\frac{3}{4} \pi-\operatorname{ph}\left(\left[p^{\prime \prime}\left(w^{(1)}\right)\right]^{\frac{1}{2}}\right)=\frac{3}{4} \pi
$$

and

$$
-\frac{3}{4} \pi-\operatorname{ph}\left(\left[p^{\prime \prime}\left(w^{(-1)}\right)\right]^{\frac{1}{2}}\right)=\frac{1}{4} \pi,
$$

that is, the square roots must be chosen so that

$$
\operatorname{ph}\left(\left[p^{\prime \prime}\left(w^{(1)}\right)\right]^{\frac{1}{2}}\right)=0, \quad \operatorname{ph}\left(\left[p^{\prime \prime}\left(w^{(-1)}\right)\right]^{\frac{1}{2}}\right)=-\pi,
$$

modulo $2 \pi$. Hence, from (17),

$$
a_{r}=\frac{-\mathrm{i}}{\sqrt{2 \pi}} \frac{\Gamma(r)}{(-2 \pi \mathrm{i})^{r}}\left[1+\mathrm{O}\left(\frac{1}{r}\right)\right]+\frac{\mathrm{i}}{\sqrt{2 \pi}} \frac{\Gamma(r)}{(2 \pi \mathrm{i})^{r}}\left[1+\mathrm{O}\left(\frac{1}{r}\right)\right]
$$

as $r \rightarrow \infty$. The result (46) simplifies to

$$
a_{r}=\sqrt{\frac{2}{\pi}}(-1)^{\frac{r-1}{2}} \frac{\Gamma(r)}{(2 \pi)^{r}}\left[1+\mathrm{O}\left(\frac{1}{r}\right)\right]
$$

as $r \rightarrow \infty$ when $r$ is odd; when $r$ is even, the contributions in (46) cancel at leading order. With account taken of the higher-order terms given in (18), one finds

$$
a_{r}=-\frac{1}{3} \sqrt{\frac{\pi}{2}}(-1)^{\frac{r}{2}} \frac{\Gamma(r)}{r(2 \pi)^{r}}\left[1+\mathrm{O}\left(\frac{1}{r}\right)\right]
$$

as $r \rightarrow \infty$ when $r$ is even.

There is no explicit, exact expression available in the literature for the coefficients $a_{r}$. The asymptotic expansion of $S^{(0)}(z)$ starts

$$
S^{(0)}(z) \sim \sqrt{2 \pi}\left(1+\frac{1}{12 z}+\frac{1}{288 z^{2}}-\frac{139}{51840 z^{3}}+\cdots\right),
$$

and, although the general form of $a_{r}$ is not known, very accurate approximations for $a_{r}$ when $r \gg 1$ are available [6, equation (71)] in the form

$$
\begin{array}{ll}
a_{r}=\frac{2(-1)^{\frac{r-1}{2}}}{(2 \pi)^{r+1}} \sum_{\substack{s=0 \\
s \text { even }}}^{M-2}(-1)^{\frac{s}{2}} a_{s}(2 \pi)^{s} \Gamma(r-s)+A_{M}, & \text { for } r=1,3,5, \ldots \\
\text { and } M \text { even, }
\end{array}
$$

where the truncation error $A_{M}$ can be explicitly and realistically bounded ([6, inequality (70)]). Noting that $a_{0}=\sqrt{2 \pi}$, one finds that (47) and (48) agree with (50) at leading order.

\section{An alternative approximation: inverse factorial series}

Berry and Howls showed how the representation (2) for $S^{(n)}(z)$ could be transformed into the form

$$
S^{(n)}(z)=\sum_{r=0}^{N-1} \frac{a_{r}}{z^{r}}+\frac{1}{2 \pi \mathrm{i} z^{N}} \sum_{m} \frac{1}{\left[p^{(n m)}\right]^{N}} \int_{0}^{\infty} \frac{v^{N-1} \mathrm{e}^{-v} S^{(m)}\left(v / p^{(n m)}\right)}{1-v / z p^{(n m)}} \mathrm{d} v
$$


[2, equation (19)]. Here, the sum is taken over the adjacent saddles; the functions $S^{(m)}$ are defined in an analogous manner to (2), but are associated with the saddle point $w^{(m)}$. In [5] we showed that the result (51) was valid provided Conditions 2.1 were satisfied with $r=N$ (and provided the integral $S^{(n)}$ actually existed).

Suppose now we put $N+1$ in place of $N$ in (51), and consider the difference between the two right-hand sides. One immediately infers that

$$
a_{r}=\frac{1}{2 \pi \mathrm{i}} \sum_{m} \frac{1}{\left[p^{(n m)}\right]^{r}} \int_{0}^{\infty} v^{r-1} \mathrm{e}^{-v} S^{(m)}\left(v / p^{(n m)}\right) \mathrm{d} v
$$

where we have written $r$ in place of $N$. This is a second representation for $a_{r}$, different from (6) or (7). Our derivation of this representation has similarities with that given by Olde Daalhuis and Olver [10] in arriving at their Lemma 3.2 (the connection is discussed further in $\S 5$ ). In many circumstances, one expects that the functions $S^{(m)}$ themselves have asymptotic expansions

$$
S^{(m)}(z) \sim \sum_{s=0}^{\infty} \frac{a_{s}^{(m)}}{z^{s}}
$$

similar to (4), as $|z| \rightarrow \infty$ for some suitably restricted range of $\mathrm{ph}(z)$. Now if $r \gg 1$, only large values of $v$ contribute significantly to the integrals in (52). Consequently, one may reasonably anticipate the use of (53) to be appropriate, and so infer the formal result

$$
a_{r} \sim \frac{1}{2 \pi \mathrm{i}} \sum_{m} \sum_{s=0}^{\infty} \frac{\Gamma(r-s)}{\left[p^{(n m)}\right]^{r-s}} a_{s}^{(m)}
$$

(cf. [2, equation (21)]). Series expansions of this kind are referred to in the literature as inverse factorial series (see $[10,12]$ and $[6, \S 3.3]$ ). We note that since

$$
a_{0}^{(m)}=\sqrt{\frac{2 \pi}{p^{\prime \prime}\left(w^{(m)}\right)}} q\left(w^{(m)}\right),
$$

the leading-order terms in (54) and (17) agree.

The result (54) is formal only. One could prove its validity rigorously by first truncating the various series in (53) and bounding their remainders, thence the remainder in the corresponding truncated version of (54). In [6] we showed how this could be done for the late coefficients in the asymptotic expansion of the gamma function. Here we shall illustrate the process by considering the late coefficients in the asymptotic expansion of the Airy function. From [5, equation (53)], one readily infers that the representation (52) is, in this case,

$$
a_{r}=\frac{1}{2 \pi \mathrm{i}(-2)^{r}} \int_{0}^{\infty} v^{r-1} \mathrm{e}^{-v} S^{(2)}\left(-\frac{1}{2} v\right) \mathrm{d} v .
$$

Now [5, equations (55), (49), (60)],

$$
S^{(2)}\left(-\frac{1}{2} v\right)=\mathrm{i} S^{(1)}\left(\frac{1}{2} v\right)=\mathrm{i} \sum_{s=0}^{M-1} 2^{s} \frac{a_{s}}{v^{s}}+R_{M}
$$

where

$$
\left|R_{M}\right| \leq \frac{2^{M}\left|a_{M}\right|}{v^{M}}
$$


Therefore, we find that

$$
a_{r}=\frac{(-1)^{r}}{2 \pi} \sum_{s=0}^{M-1} \frac{\Gamma(r-s)}{2^{r-s}} a_{s}+A_{M}
$$

where

$$
\left|A_{M}\right| \leq \frac{1}{2 \pi} \frac{\Gamma(r-M)}{2^{r-M}}\left|a_{M}\right|
$$

One readily establishes that the least value of $\left|A_{M}\right|$ occurs when $M \approx \frac{1}{2} r$. At leading order, (59) agrees with our earlier result (27) (noting that, from (25), $a_{0}=\mathrm{i} \sqrt{2 \pi / 3}$ ).

One may likewise show that for the modified Bessel function,

$$
a_{r}=\frac{(-1)^{r}}{\pi} \cos (\nu \pi) \sum_{s=0}^{M-1} \frac{\Gamma(r-s)}{2^{r-s}} a_{s}+A_{M}
$$

where

$$
\left|A_{M}\right| \leq \frac{\cos (\nu \pi)}{\pi} \frac{\Gamma(r-M)}{2^{r-M}}\left|a_{M}\right|
$$

\section{Discussion}

Many results concerning the behavior of late coefficients in asymptotic expansions are given in the book by Dingle [9]. Dingle's derivation of his results is based on interpretive, rather than rigorous, methods. The analysis which we have provided in this paper provides a secure basis for investigation of some of the questions which he addresses.

In particular, the issues considered in Chapters VII and VIII of [9] (late terms in general and in integral representations, respectively) can be tackled directly. Dingle based his derivations on Darboux's theorem [9, p. 141], but he expressed dissatisfaction with merely taking account of "the singular points on the circle of convergence", as the theorem requires; instead, he advocated expanding "about every singularityboth on and outside the circle of convergence". From our current standpoint-with the advantage provided by the reformulation of the method of steepest descents due to Berry and Howls [2]-we can express this more precisely as follows. The analogue of the Darboux theorem in our approach is that only those adjacent saddle points which share the least value of $\left|p^{(n m)}\right|$ contribute to the asymptotic estimate for $a_{r}$ as $r \rightarrow \infty$, (cf. equation (19)). However, there is a definite sense in which it is proper to consider also the contributions from other saddle points; the coefficient $a_{r}$ is represented exactly by a sum of terms, each of which is strongly associated with the local behavior at the adjacent saddle points (cf. equations (10) and (52)). We remark that the set of adjacent saddle points may be smaller than the set of all other saddle points, so that Dingle's advocacy needs to be interpreted subject to this proviso.

The results which we have obtained in $\S 4$ may be compared directly (for the Airy function and for the modified Bessel function) with those which have been found by Olver [12] and Olde Daalhuis and Olver [10]. Olver's approach is based on exploiting the recurrence relation enjoyed by the coefficients $a_{r}$, while Olde Daalhuis and Olver exploited the integral representation (via Cauchy's integral formula) of solutions of linear second-order differential equations. Their results are consistent with ours, though we should remark that we have explicitly identified all of the constants used and have obtained explicit error bounds.

It is of interest to note the generality of the results (17) and (19); the behavior of the late coefficient $a_{r}$ in the asymptotic expansion arising from the method of steepest 
descents can be expected to be in the form $\Gamma(r) / p^{r}$, or a modification of this form as in the case of the gamma function. This behavior of 'a factorial divided by a power' was used to considerable effect by Dingle [9, p. 405] as the basis for the formal development of his theory of terminants and by Berry [1] in his explanation of the Stokes phenomenon. The class of integrals which we have considered in this paper is a fairly wide one; consequently our discussion provides some insights into the reasons for the success of their formal methods. It should be noted that the assumptions which we made about the properties of $p(w)$ (Conditions 2.1) are less restrictive than might first appear; it often is possible to make a preliminary change of integration variable to bring the integrand into the appropriate form. (In effect, this is what we did in the case of the gamma function in transforming from (38) to (39).)

\section{Appendix}

The purpose of this appendix is to demonstrate the equivalence of apparently inconsistent representations for the coefficients $a_{r}$ in the asymptotic expansion of the gamma function, which we considered in $§ 3.3$. The representation for the coefficients which we implicitly used in $\S 3.3$ was

$$
a_{r}=\frac{\Gamma\left(r+\frac{1}{2}\right)}{2 \pi \mathrm{i}} \oint_{\Gamma^{(0)}} \frac{1}{\left[\mathrm{e}^{w}-w-1\right]^{r+\frac{1}{2}}} \mathrm{~d} w
$$

where the contour $\Gamma^{(0)}$ surrounds the saddle point $w^{(0)}=0$.

Diekmann, in effect, considers the expression

$$
a_{r}=\frac{\Gamma(2 r+1) \sqrt{\pi}}{\Gamma(r+1) 2^{2 r}} \frac{1}{2 \pi \mathrm{i}} \oint \frac{1}{[z-\ln (1+z)]^{r+\frac{1}{2}}} \mathrm{~d} z
$$

for the coefficients [8, equations (2.1)-(2.3), (2.5), (3.2)]. (The definition of the coefficients used by Diekmann differs from that used in $\S 3.3$ of this paper by a factor of $\sqrt{2 \pi}$.) The contour of integration in (64) is a closed contour surrounding $z=0$. The transformation $1+z=\mathrm{e}^{w}$, together with the duplication formula for the gamma function [11, p. 35], yields

$$
a_{r}=\frac{\Gamma\left(r+\frac{1}{2}\right)}{2 \pi \mathrm{i}} \oint_{\Gamma^{(0)}} \frac{\mathrm{e}^{w}}{\left[\mathrm{e}^{w}-w-1\right]^{r+\frac{1}{2}}} \mathrm{~d} w
$$

where $\Gamma^{(0)}$ is defined as in (63) above. The result (65) is similar in form to (63), but is nevertheless not identical with it.

It is worth confirming that (63) and (65) are both correct, and that both may be derived in the same manner. By appealing to Euler's integral (38) for $\Gamma(z+1)$ and the identity $\Gamma(z+1) / z=\Gamma(z)$, one finds that

$$
\Gamma(z)=z^{z} \int_{-\infty}^{\infty} \mathrm{e}^{-z p(w)} q(w) \mathrm{d} w
$$

where $p(w)$ is defined as in (40) and $q(w)=\mathrm{e}^{w}$. Using (66) in place of (39) leads directly to the representation (65) in place of (63). So, despite the difference between the integrands in (63) and (65), both are valid.

\section{References}

1. M. V. Berry, Uniform asymptotic smoothing of Stokes's discontinuities, Proc. R. Soc. Lond. A 422 (1989), 7-21.

2. M. V. Berry and C. J. Howls, Hyperasymptotics for integrals with saddles, Proc. R. Soc. Lond. A 434 (1991), 657-675. 
3. __ Unfolding the high orders of asymptotic expansions with coalescing saddles: singularity theory, crossover and duality, Proc. R. Soc. Lond. A 443 (1993), 107-126.

4. W. G. C. Boyd, Stieltjes transforms and the Stokes phenomenon, Proc. R. Soc. Lond. A $\mathbf{4 2 9}$ (1990), 227-246.

5. _ Error bounds for the method of steepest descents, Proc. R. Soc. Lond. A 440 (1993), 493-518.

6. - Gamma function asymptotics by an extension of the method of steepest descents, Proc. R. Soc. Lond. A 447 (1994), 609-630.

7. E. T. Copson, Asymptotic Expansions, Cambridge University Press, Cambridge, 1965.

8. O. Diekmann, Asymptotic expansion of certain numbers related to the gamma function, Report TN 80/75, Stichting Mathematisch Centrum, Amsterdam, 1975.

9. R. B. Dingle, Asymptotic Expansions: Their Derivation and Interpretation, Academic Press, London, 1973.

10. A. B. Olde Daalhuis and F. W. J. Olver, Exponentially-improved asymptotic solutions of ordinary differential equations II: irregular singularities of rank one, Proc. R. Soc. Lond. A 445 (1994), 39-56.

11. F. W. J. Olver, Asymptotics and Special Functions, Academic Press, New York, 1974.

12. __ Asymptotic expansions of the coefficients in asymptotic series solutions of linear differential equations, Methods and Applications of Analysis, 1 (1994), 1-13.

13. R. Wong, Asymptotic Approximations of Integrals, Academic Press, New York, 1989.

School of Mathematics, University of Bristol, Bristol BS8 1TW, United Kingdom 